\title{
Tri-vector boson production at NLO with parton shower
}

\author{
M.K. Mandal \\ Regional Centre for Accelerator-based Particle Physics \\ Harish-Chandra Research Institute, Chhatnag Road, Jhunsi, \\ Allahabad 211019 , India \\ E-mail: mandalehri.res.in \\ P. Mathews \\ Saha Institute of Nuclear Physics, 1/AF Bidhan Nagar, Kolkata 700 064, India \\ E-mail: prakash.mathews@saha.ac.in

\section{Ravindran*} \\ Institute of Mathematical Sciences, C.I.T. Campus, Taramani,Chennai \\ E-mail: ravindra@imsc.res.in

\section{S. Seth} \\ Saha Institute of Nuclear Physics, 1/AF Bidhan Nagar, Kolkata 700 064, India \\ E-mail: satyajit.seth@saha.ac.in
}

\begin{abstract}
We have studied the production of three photons at the LHC taking into account realistic experimental cuts. We have used NLO corrected QCD results for the production mechanism along with parton showering through HERWIG and Pythia. This is achieved using aMC@NLO framework which uses FKS subtracton method through MadFKS to handle infrared singularities present in the intermediate stages of NLO computation. We find that the results at NLO level in QCD with parton showering show less PDF and factorisation scale dependence.
\end{abstract}

11th International Symposium on Radiative Corrections (Applications of Quantum Field Theory to Phenomenology)

22-27 September 2013

Lumley Castle Hotel, Durham, UK

${ }^{*}$ Speaker. 


\section{Introduction}

Lepton, gauge boson and hadron productions at the Tevatron and at the Large Hadron Collider (LHC) provide ample opportunity not only to test the predictions of the Standard Model (SM) but also to constrain various physics scenario of beyond the standard model (BSM). The later are subjected to large standard model background and hence careful and precise predictions of wide variety of SM processes have been underway [1]. Such predictions for SM processes are important as the quantum corrections are comparable to the BSM effects. Also, they suffer from large theoretical uncertainties making the leading order (LO) predictions unreliable. One source of uncertainty is from the factorisation scale $\mu_{F}$ that is introduced to remove collinear divergences coming from initial state massless partons. This necessitates the computation of next-to-leading order (NLO) quantum effects from Quantum Chromodynamics (QCD). The choice of parton density functions will also introduce uncertainty in the predictions and we will present their impact also. The realistic predictions from the theory is another issue which we have to deal with in order to compare against the data. Here parton level predictions need to go through parton showering (PS) and recombination of partons into hadrons through hadronization mechanism. Such predictions require careful matching to avoid double counting. Here, we revisit the NLO QCD results for the production of three photons in hadron collisions in NLO+PS accuracy in order to get realistic estimates for various distributions. This process has been already studied at LO [2,3] as well as NLO level [4] in QCD. We quantify the improvement in the predictions at small transverse momentum regions of the final states and also the stabilisation of cross section against factorisation and renormalisation scales.

\section{NLO+PS computation}

Leading order $\left(\mathscr{O}\left(\alpha^{3}\right)\right)$ contributions to production of three photons at hadron colliders result from purely quark anti-quark annihilation processes. At NLO $\mathscr{O}\left(\alpha^{3} \alpha_{s}\right)$ in $\mathrm{QCD}$, both virtual as well as real emission contributions due to an additional parton, namely quark or anti-quark or gluon will contribute. Virtual amplitudes are at $\mathscr{O}\left(\alpha^{3 / 2} \alpha_{s}\right)$, hence the interference of them with the LO Born amplitudes will contribute at NLO level. The real emission processes include gluon emissions from the leading order processes and scattering of a quark (anti-quark) and a gluon producing three photons along with a quark (antiquark). The ultraviolet divergences from the virtual contributions are removed by proper counter terms and the infra-red divergences present in both virtual and real emission processes are removed by mass factorisation.

$$
\begin{aligned}
d \hat{\sigma}_{a b}^{N L O}= & \int d P S_{3 \gamma} S\left(\{p\}_{1,5}\right) d \hat{\sigma}_{a b}^{(0)} \\
& +\frac{\alpha_{s}\left(\mu_{R}\right)}{4 \pi}\left[\int d P S_{3 \gamma} S\left(\{p\}_{1,5}\right) d \hat{\sigma}_{a b}^{V,(1)}+\int d P S_{3 \gamma} S\left(\{p\}_{1,5}\right) d \hat{\sigma}_{a b}^{C T,(1)}\right. \\
& \left.+\int d P S_{3 \gamma+\text { parton }} S\left(\{p\}_{1,6}\right) d \hat{\sigma}_{a b}^{R,(1)}+\int d P S_{3 \gamma} S\left(\{p\}_{1,5}\right) d \hat{\sigma}_{a b}^{M F,(1)}\right]
\end{aligned}
$$

The first line in the above equation is the Born contribution, $d P S_{3 \gamma}$ is the phase space measure for the three photon final state and $S\left(\{p\}_{1, m}\right)$ is the observable function which depends on the 
kinematic variables. The second line corresponds to virtual contributions to Born processes and the corresponding counter terms. The real emission contributions at the NLO level that come from parton emissions from the initial and final state partons suffer from soft and collinear divergences due to their massless nature. The divergences coming from soft gluons and from collinear partons in the final state of the real emission processes cancel with those coming from virtual processes. The remaining collinear divergences from the initial states are removed by adding mass counter terms given in the last line. We have used MadFKS ([5]) to obtain real emission contributions and their mass counter terms. A set of routines available in the aMC@NLO [6], which along with our FORTRAN routines for virtual contributions can provide results on an event-by-event basis in terms of four momenta of all the particles involved in the scattering event and its associated weight. We use them to obtain the observables that we require to study.

There are forty eight virtual amplitudes to order $\alpha^{3 / 2} \alpha_{s}$. Upto permutations of the final state photons, we find one pentagon diagram, 2 box diagrams, 3 triangle diagrams and 2 bubble diagrams. We use QGRAF [7] to generate these amplitudes, and in-house FORM [8] code could translate the output of QGRAF into a form that can be used for further symbolic manipulations. We have supplied relevant Feynman rules, various identities for Dirac gamma matrices, equations of motion and performed simplifications at the amplitude level. We have used dimensional regularisation and the Lorentz contractions and Dirac gamma matrix simplifications are done in $n=4+\varepsilon$ space time dimensions. Both ultraviolet and infra-red divergences show up as poles in $\varepsilon$ and they are removed through UV renormalisation and mass factorisation respectively. We write the virtual contribution as

$$
\sum_{\text {col spin }} \mathscr{M}^{V,(1)}\left(\mathscr{M}^{(0)}\right)^{*}=\sum_{\Gamma}\left[\sum_{\text {col spin }} \mathscr{M}^{V,(\Gamma)}\left(\mathscr{M}^{(0)}\right)^{*}\right]
$$

where $\mathscr{M}^{(0)}$ is the born amplitude and $\mathscr{M}^{V,(\Gamma)}$ are the distinct topologies of virtual diagrams.

The reduction of tensor integrals to scalar ones in $n$ dimensions is done using PassarinoVeltman reduction. We have written a FORM code to do this reduction and expressed the virtual contributions in terms of scalar coefficients. These coefficients are related to scalar integrals in higher dimensions. Following ref ([9]), these integrals can be written in terms of integrals in $n$ dimensions thanks to dimensional recurrence relations discussed in [10]. The problem with inverse Gram determinants that result from the recurrence relations often spoil the numerical stability of the integrals can be dealt with using an elegant approach that has been put forward in [11] where the authors have found signed minor algebraic relation which avoids these inverse determinants. This does not reduce the shifted dimension to $n$. In ref. [11], the computation of these additional shifted integrals are done numerically after employing a series expansion in the small Gram region. This has been implemented in PJFry package [12], which we use to evaluate the scalar coefficients numerically for every phase space point in $n$ dimensions. It uses QCDLoop [13] and OneLOop [14] to evaluate the scalar integrals in 4 dimensions. Using our FORM codes and FORTRAN routines along with the publicly available packages namely QGRAF, PJFry and QCDLoop/OneLOop, we have evaluated virtual contributions to 3 photon production at $\alpha_{s}$ level. We find that after UV renormalisation, the infra-red poles namely double and single poles in $\varepsilon$ are in accordance with the expectation. We express the virtual contribution to 3 photon production in the form suitable for 
further analysis:

$$
d \hat{\sigma}_{q \bar{q}}^{V,(1)}=\frac{\alpha_{s}}{2 \pi} \frac{1}{\Gamma\left(1+\frac{\varepsilon}{2}\right)}\left(\frac{s}{4 \pi \mu_{R}^{2}}\right)^{\frac{\varepsilon}{2}} C_{F}\left(-\frac{8}{\varepsilon^{2}}+\frac{6}{\varepsilon}\right) d \hat{\sigma}_{q \bar{q}}^{(0)}+d \hat{\sigma}_{q \bar{q}}^{V,(1), f i n}\left(\mu_{R}\right)
$$

where $\mu_{R}$ is renormalisation scale, the colour factors $C_{F}=4 / 3$ for $S U(3) . d \hat{\sigma}_{q \bar{q}}^{(0)}$ comes from Born amplitude.

\section{Numerical implementation}

We use aMC@NLO [6] framework not only to compute real emission contributions along with the mass counter terms but also to obtain parton showered results. Within aMC@NLO, MadGraph generates all the required tree level matrix elements upto NLO level. MadFKS [5] separates out soft and collinear configurations in the real emission processes using the subtraction scheme proposed by Frixione, Kunzst and Signer [15], namely FKS subtraction scheme and provides IR divergent and IR safe contributions as well as the mass counter terms to remove the initial state collinear divergences. The program provides the IR pole values which will then be cancelled against those coming from the virtual part. We have explicitly checked cancellation of the soft and collinear divergences among virtual, real and mass counter terms at large number phase space points for various choices of $S\left(p_{i}\right)$. Then, the hard and standard events are generated in the aMC@NLO using MINT/MINTMC MonteCarlo. The generated events are then showered by Herwig [16] and Pythia [17] to get the realistic events.

It is well known fact that photons are produced not only at the partonic level but also through fragmentation of partons into a photon and a jet of hadrons, often collinear to it. They introduce non-perturbative fragmentation functions in the theoretical framework. At NLO level, this QED collinear divergence arises when one of the final state partons becomes collinear to a photon. They can be again factorised in universal manner and removed by additional mass counter terms which renormalise the fragmentation functions. This introduces fragmentation scale in the perturbative results. The fragmentation functions and the new scale give additional uncertainties. Hence, it is desirable to avoid the introduction of non-perturbative fragmentation functions and also the associated fragmentation scale. Smooth cone isolation provides the alternative. The fragmentation contributions will be absent if we do not allow QED collinear configurations in the final state in a infra-red safe way. This can be done if we remove only hard partons that are collinear to photon. In [18], Frixione proposed an elegant isolation criteria which gives an observable where fragmentation contribution is minimised, at the same time the infra-red safety is guaranteed. This demands that the sum of hadronic transverse energy inside any concentric circle of radius $R<R_{0}$ in the $\theta-\phi$ plane be less than an amount given by a function $H(R)$ which goes to zero as $R \rightarrow 0$. For our analysis, we have taken the following canonical choice for $H(R)$, namely

$$
H(R)=E_{T}^{i s o}\left(\frac{1-\cos R}{1-\cos R_{0}}\right)^{n}
$$

where $E_{T}^{i s o}$ is a fixed energy. 


\section{Results}

We next present the results for few kinematic distributions relevant to the production of three photons in SM at the $14 \mathrm{TeV}$ LHC. The input parameters used are

$$
\begin{aligned}
M_{W} & =80.419 \mathrm{GeV}, \quad \sin ^{2} \theta_{W}=0.222, \\
M_{Z} & =91.188 \mathrm{GeV}, \quad \alpha_{e m}^{-1}=132.507, \\
G_{F} & =1.16639 \cdot 10^{-5} \mathrm{GeV}^{-2} .
\end{aligned}
$$

We have considered only massless quarks and use MSTW2008(N)LO parton distribution functions with errors estimated at $68 \%$ for the $(\mathrm{N}) \mathrm{LO}$ and it also sets the value of the strong coupling $\alpha_{s}\left(M_{Z}\right)$ at LO and NLO in QCD. The factorisation scale $\left(\mu_{F}\right)$ and the renormalisation scale $\left(\mu_{R}\right)$ are set equal to a central scale $\left(\mu_{0}\right)$, which is the invariant mass of the three photon final state i.e. $\mu_{F}=\mu_{R}=\mu_{0}=M_{\gamma \gamma \gamma} \equiv \sqrt{\left(P_{\gamma_{1}}+P_{\gamma_{2}}+P_{\gamma_{3}}\right)^{2}}$. For the fixed order (N)LO calculation, two choices of cuts are used. CUTI: transverse momentum of the photons $P_{T}^{\gamma}>20 \mathrm{GeV}$, rapidity $\left|\eta^{(\gamma)}\right|<2.5$, the separation of the two photons in the rapidity-azimuthal plane $\Delta R^{\gamma \gamma}>0.4$, where $\Delta R^{\gamma \gamma}=\sqrt{(\Delta \eta)^{2}+(\Delta \phi)^{2}}$ and Frixione isolation with the parameters $R_{0}=0.7, \varepsilon_{\gamma}=1$ and $n=2$ and CUTII: $P_{T}^{\gamma}>30 \mathrm{GeV},\left|\eta^{(\gamma)}\right|<2.5, \Delta R^{\gamma \gamma}>0.4$ and Frixione isolation with the parameters $R_{0}=0.7$, $\varepsilon_{\gamma}=1$ and $n=2$,. We use the following loose cuts when the parton level events are generated: $P_{T}^{(\gamma)}>15 \mathrm{GeV},\left|\eta^{(\gamma)}\right|<2.7, \Delta R^{\gamma \gamma}>0.3$, Frixione isolation with $R_{0}=0.6, \varepsilon_{\gamma}=1$ and $n=2$. We have used HERWIG6 (HW6) and PYTHIA6 (PY6) to shower the events and then actual analysis cuts are used along frixione isolation. We have taken the cuts at the analysis level as CUTII: $P_{T}^{\gamma}>30 \mathrm{GeV},\left|\eta^{(\gamma)}\right|<2.5, \Delta R^{\gamma \gamma}>0.4$ and Frixione isolation with $R_{0}=0.7, \varepsilon_{\gamma}=1$ and $n=2$. We have estimated the scale dependence by varying the factorisation scale $\left(\mu_{F}\right)$ and renormalisation scale $\left(\mu_{R}\right)$ independently around the central value $\mu_{F}=\mu_{R}=M_{\gamma \gamma \gamma}$ via the following assignment $\mu_{F}=\xi_{F} M_{\gamma \gamma \gamma}$ and $\mu_{R}=\xi_{R} M_{\gamma \gamma \gamma}$, where $\xi_{F}$ and $\xi_{R}$ varies between the range [1/2,2] independently. The scale uncertainty band is the envelope of the results obtained by varying this $\xi_{F}$ and $\xi_{R}$ within this range.

The PDF uncertainties are estimated with the hessian method, as given by MSTW [19] set. We have plotted fractional uncertainty, which is defined as the ratio of the variation about the central value divided by the central value, being a good indicator of the uncertainties. These uncertainty bands can be obtained at no extra CPU cost within the aMC@NLO framework as described in Ref. [20].

We now present our numerical results for the distributions of transverse momentum $p_{T}^{\gamma}$ of the hardest photon in the left panel of Fig.(1) and the invariant mass $M^{\gamma \gamma \gamma}$ of the three photons in the right panel of fig(4.1) using our fixed order NLO as well as the NLO matched with PS (NLO+PS) results for two different choices of showers namely HW6 and PY6. At low $p_{T}^{\gamma}$, NLO+PS (for both HW6 and PY6) resums the Sudakov logarithms leading to a suppression of the cross section while the fixed order NLO results tend to diverge. At high enough $p_{T}^{\gamma}$, the NLO fixed order and NLO+PS (for both HW6 and PY6) results coincide as expected. In the upper and lower insets of the Fig.(4.1), we have shown the sensitivity of our predictions to the scale and PDF choices using the NLO+PS results. The PDF uncertainty is moderate for the entire range for both the distributions. But the scale uncertainty in the transverse momentum distribution shows dependence on the $p_{T}^{\gamma}$ 
and it is small in the small $p_{T}^{\gamma}$ region due to Sudakov resummation in parton showering. Large $p_{T}^{\gamma}$ is fully controlled by fixed order NLO result which has explicit renormalisation scale that will be compensated only NNLO level, hence there is a strong dependence on $\mu_{R}$ in this region. We have also found (not given in the plot separately) that factorisation scale dependence goes down significantly if we include NLO correction as expected.
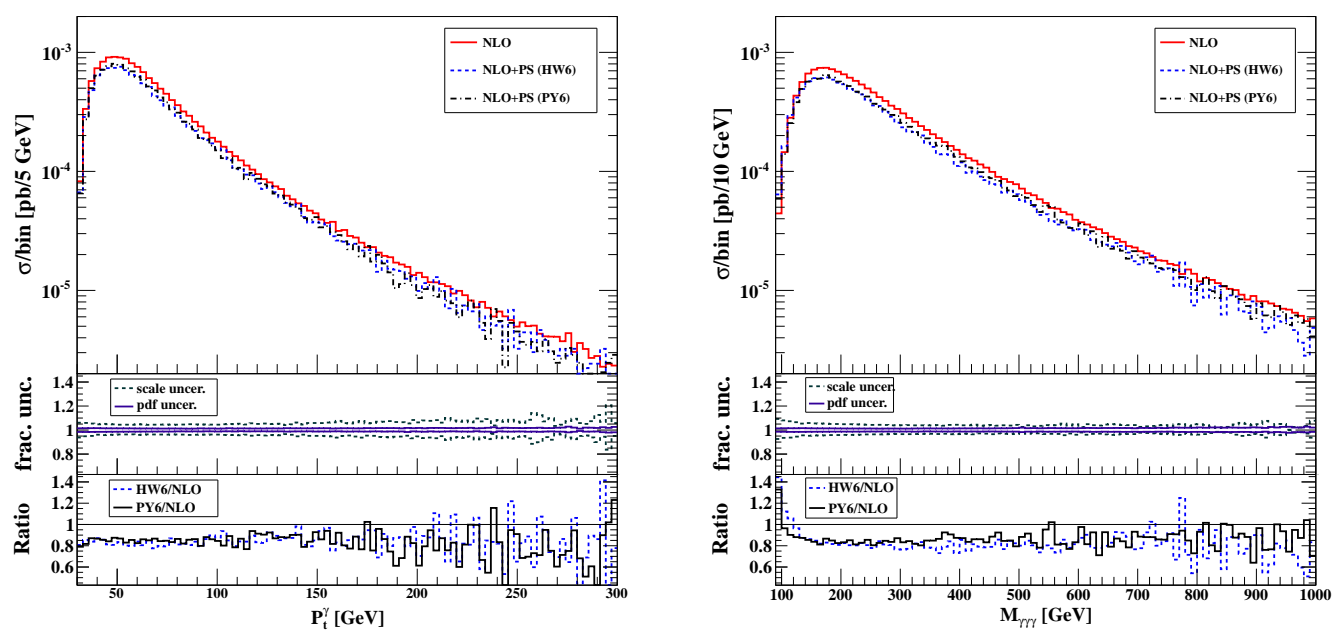

Figure 1: Transverse momentum distribution $p_{T}^{\gamma}$ of the hardest photon among the photons for the fixed order NLO and NLO+PS for HW6 and PY6 (left panel) and invariant mass distribution $M_{\gamma \gamma \gamma}$ of the three photons for the fixed order NLO and NLO+PS for HW6 and PY6 (right panel).

\section{Conclusion}

Precise and realistic predictions of both signal and background processes at hadron colliders are now possible due to various important developments in the computational methods and the availability of state of the art computational tools. We have used packages like PJFry, aMC@ NLO and several other computer codes listed in this article to study three photon production at the NLO level in QCD for the LHC taking into account parton showering and realistic experimental cuts. We find our predictions are less sensitive to factorisation scale and choice of PDFs and hence more suited for direct comparison with the data from the experiments.

\section{Acknowledgements}

M.K.M and V.R would like to thank Regional Centre for Accelerator-based Particle Physics (RECAPP) at Harish-Chandra Research Institute, Allahabad for financial support during the course of this work. V.R would like to thank the organisers of RADCOR for local hospitality during the symposium. 


\section{References}

[1] J.M. Campbell, J.W. Huston, W.J. Stirling, Rep. Prog. Phys. 70 (2007) 89, The SM and NLO Multileg Working Group: Summary report, J. R. Andersen et.al., Mar 2010, The SM and NLO Multileg and SM MC Working Groups,: Sumary Report, 1203.6803.

[2] M. Golden, S.R. Sharpe, Nucl. Phys. B261 (1985) 217, V. Barger, T. Han, Phys. Lett. B212 (1988) 117.

[3] M. C. Kumar, P. Mathews, V. Ravindran and S. Seth, Phys. Rev. D 85 (2012) 094507 [arXiv:1111.7063 [hep-ph]].

[4] T. Binoth, G. Ossola, C.G. Papadopoulos, R. Pittau, JHEP 0806 (2008) 082, A. Lazopoulos, K. Melnikov, F. Petriello, Phys. Rev. D76 (2007) 014001, G. Bozzi, F. Campanario, V. Hankele, D. Zeppenfeld, Phys. Rev. D81 (2010) 094030, G. Bozzi, F. Campanario, M. Rauch, D. Zeppenfeld, Phys. Rev. D84 (2011) 074028.

[5] R. Frederix, S. Frixione, F. Maltoni and T. Stelzer, JHEP 10 (2009) 003.

[6] R. Frederix, S. Frixione, V. Hirschi, F. Maltoni, R. Pittau and P.Torrielli, Phys. Lett. B701 (2011) 427; R. Frederix, S. Frixione, V. Hirschi, F. Maltoni, R.Pittau and P. Torrielli, JHEP 09 (2011) 061.

[7] P. Nogueira, Journal of Computational Physics 105 (1993) 279.

[8] M.Tentyukov and J.A.M. Vermaseren, hep-ph/0702279.

[9] A. I. Davydychev, Phys. Lett. B263 (1991) 107.

[10] O.V. Tarasov, Phys. Rev. D54 (1996) 6479, J. Fleischer, F.Jegerlehner, and O.V. Tarasov, Nucl. Phys. B566 (2000) 423.

[11] J. Fleischer and T. Riemann, Phys. Rev. D83 (2011) 073004.

[12] V. Yundin, Ph.D thesis, Humboldt-Universitat zu Berlin, 2012, http://edoc.hu-berlin.de/docviews/abstract.php?id=39163;https://github.com/Vayu/PJFry/.

[13] R.K. Ellis and G. Zanderighi, JHEP 02 (2008) 002.

[14] A. van Hameren, Comput. Phys. Commun. 182 (2011) 2427.

[15] S. Frixione, Z. Kunszt and A. Signer, Nucl. Phys. B467 (1996) 399, S. Frixione, Nucl. Phys. B507 (1997) 295

[16] G. Marchenini et al., HERWIG, Comput. Phys. Commun. 67 (1992) 465, G. Corcella et al., HERWIG 6.5, JHEP 01 (2001) 010, G. Corcella et al., HERWIG 6.5 release note, hep-ph/0210213.

[17] T. Sjostrand, S. Mrenna and P. Z. Skands, JHEP 0605 (2006) 026 [hep-ph/0603175].

[18] S. Frixione, Phys.Lett. B429 (1998) 369.

[19] A. D. Martin, W. J. Stirling, R. S. Thorne, and G. Watt, Eur. Phys. J. C63 (2009) 189âĂŞ285.

[20] R. Frederix, S. Frixione, V. Hirschi, F. Maltoni, R. Pittau and P. Torrielli, JHEP 02 (2012) 099. 